\title{
Digital telemetry transmitter in Crew Escape System
}

\author{
K. S. Smitha*, Sherly Joy, D. Sheba Elizabeth, Sathya Bhushan Shukla, \\ S. Sanoj Kumar Roy and K. K. Mukundan \\ Vikram Sarabhai Space Centre, Indian Space Research Organisation, Thiruvananthapuram 695 022, India
}

\begin{abstract}
This article presents an efficient digital telemetry transmitter with Offset Quadrature Phase Shift Keying (OQPSK) modulation for launch vehicle applications with direct modulation in RF carrier. Analog transmitters with phase modulation are widely used for data transmission in launch vehicles. To gain the advantage of digital modulation like bandwidth efficiency and power efficiency, a digital telemetry transmitter with OQPSK modulation and half rate Turbo coding was flown in ISRO's Human Space Programme Pad Abort Test Crew Escape System. In OQPSK, compared to conventional QPSK, the quadrature channel bits lag the in-phase channel bits by one bit period. This feature is an advantage of the OQPSK modulation technique as it overcomes the $180^{\circ}$ phase transitions, eliminating the possibility of crossing through the origin. Thus it provides a narrow power spectrum with less inter-symbol interference. This transmitter is employed with direct modulation in $\mathrm{S}$-band $\mathrm{RF}$ carrier as it provides a less complex and simple architecture compared to the conventional IF modulation and up-conversion techniques.
\end{abstract}

Keywords: Crew Escape System, digital modulation, launch vehicles, telemetry, transmitter.

\section{Introduction}

IN launch vehicles (LVs), RF link is the sole communication available for establishing data transmission to the ground. It is utilized to transmit information within LV like health parameters of different subsystems, scientific data, images/videos of events to the ground station in real time. RF transmitters on-board LV together with its antenna system perform this function.

In LVs, telemetry data transmission is an automated communication process where vehicle data are collected, formatted, modulated and transmitted to the receiving station for data retrieval. Geographically separated India Space Research Organisation (ISRO) ground stations receive telemetry data from LV. In certain scenarios where line-of-sight constraints exists, essential data at a lesser rate, due to the larger distance involved, will be relayed

*For correspondence. (e-mail: ks_smitha@vssc.gov.in) to the ground station through a satellite in geostationary orbit. In LVs, linear analog phase modulation is employed due to its quintessential property of in-built residual carrier in the modulated output. This enables simple and easy phase modulation (PM) demodulation at the receiver end. However, it occupies more bandwidth and cannot be used for band-limited applications as it is a less bandwidth-efficient scheme. Digital modulation schemes like BPSK, QPSK, Minimum Shift Keying (MSK) offer better bandwidth efficiency when compared with the conventional counterpart - analog (phase) modulation.

This article describes the design of an efficient Offset Quadrature Phase Shift Keying (OQPSK) modulated digital telemetry transmitter with direct modulation in RF carrier. The digital transmitter is incorporated with in-built channel coding. The article also discusses the significance of transmitter in a $\mathrm{LV}$, digital transmitter design and link calculation, carrier generation techniques using PLL, IQ modulation, overview of OQPSK modulation and its advantages, and system performance in the Pad Abort Test (PAT) mission.

\section{OQPSK modulation}

In QPSK $^{1}$, the in-phase $(I)$ and quadrature phase $(Q)$ bit components are switched simultaneously. Thus the symbol clocks are synchronized. This allows the signals to pass the origin, which means the carrier amplitude dips to zero at that instant. This results in some symbols with large amplitude variations and others with small amplitude variations. It leads to uncertainty in the clock recovery subsystem of the receiver. In addition, spectral regrowth (spectral splatter) occurs if such signals are subjected to nonlinear region of amplifiers. Hence perfectly linear amplifiers are mandatory for QPSK signals ${ }^{2}$.

This difficulty can be overcome by employing $I$ and $Q$ signals offset in their relative arrangement by one bit period. It ensures that the carrier amplitude does not go through the centre (origin) of the constellation. This is possible as only one of the bit streams changes values at any time instant. It offers more advantages like better power efficiency as nonlinear power amplifier can be used without spectral regrowth or splatter ${ }^{3}$. Figure 1 shows the constellation diagrams of OQPSK and QPSK. 
OQPSK is an alternative form of QPSK in which the $Q$ channel is delayed by one bit period or half symbol period (Figure 2), so that signal transition in both the $I$ and $Q$ channels does not occur at the same time. In QPSK, the maximum phase transition can be as high as $180^{\circ}$.

Thus the band-limited QPSK system can exhibit envelope fluctuation and interfere with adjacent channels. However, due to the delay introduced in the OQPSK system, phase transition cannot exceed more than $90^{\circ}$. Due to the reduced phase transition in the OQPSK system, the spectral splatter is less when compared with the QPSK system. Figure 3 shows the block diagram of the OQPSK transmitter.

\section{Design of digital telemetry transmitter}

A $0.5 \mathrm{~W}$ OQPSK modulated digital telemetry transmitter with direct modulation on S-band RF carrier which is
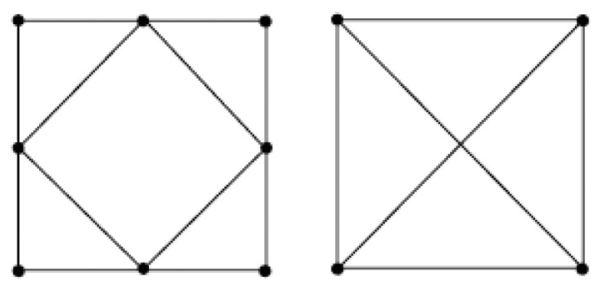

Figure 1. Constellation diagrams of OQPSK and QPSK.

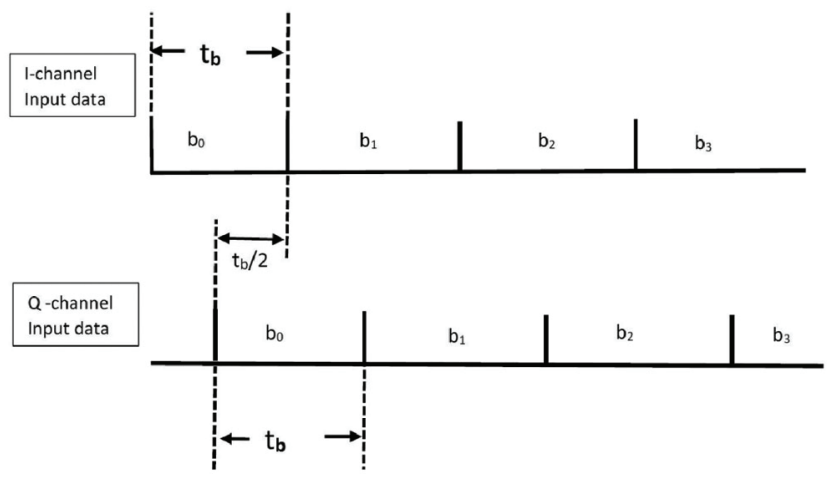

Figure 2. OQPSK signals.

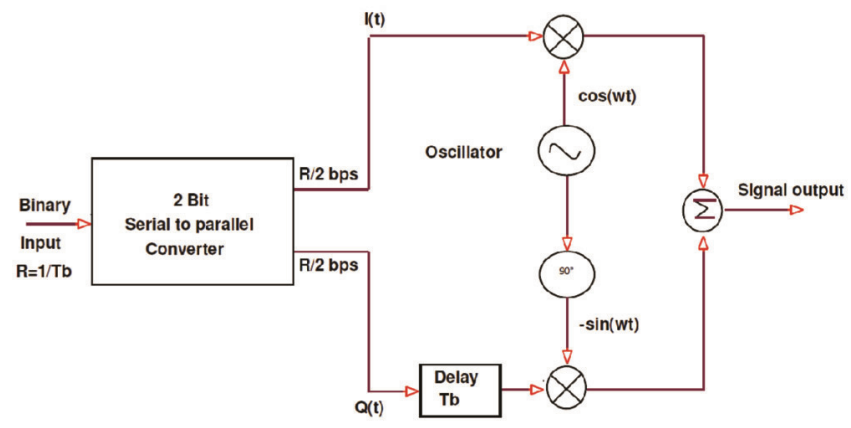

Figure 3. Block diagram of OQPSK transmitter. capable of transmitting 1 Mbps data rate was designed, developed and flown in Human Space Programme (HSP)PAT. Using digital modulation, bandwidth requirement is significantly less compared to an analog PM transmitter for the same power. Hence more data can be transmitted using a digital transmitter for a given amount of bandwidth and power with respect to its analog counterpart. OQPSK is selected based on the advantages on power and spectral efficiency, inter-symbol interference (ISI) and possibility of nonlinear operation of power amplifier.

Figure 4 shows the block diagram of the transmitter. Here, the raw message signal is half rate turbo-coded and split into offset quadrature signals $(I$ and $Q$ ) at the required rate compatible for performing OQPSK. These signals are further conditioned to perform the OQPSK digital modulation directly in the S-band RF carrier. This modulated signal is amplified to the required level and transmitted through the S-band antenna. The transmitter has the capability of operating in frequency selection mode up to $1 \mathrm{kHz}$ resolution. The transmitter output is fed to an S-band power divider. Two individual patch antennas are connected at the output ports of the power divider to feed two antennas. The two patch antennas together provide omni-directional coverage. The system works with an in-built DCDC converter and it provides the DC power requirement of the subsystems of the transmitter (modulator and amplifiers) and the baseband system. The DC/DC converter tackles power supply variation from 24 to $36 \mathrm{~V}$ DC. An EMI filter is also included in the power supply circuit to manage the electromagnetic interference according to MIL-STD-461 \& 462.

\section{Specifications of transmitter}

The specifications of the transmitter are given below.

Transmitter frequency: $2.2-2.3 \mathrm{GHz}$

Power output: $0.5 \mathrm{~W}$ min

Modulation: OQPSK

Symbol rate: 1 Mbps (half rate turbo-coded) in $I$ and $Q$ Carrier suppression: Better than $25 \mathrm{~dB}$.

\section{Link calculation}

Link calculation is done based on the specifications of on-board transmitter, antenna and ground station.

Transmit frequency: $2.2-2.3 \mathrm{GHz}$

Transmit power output: $+27 \mathrm{dBm}(0.5 \mathrm{~W})$

Transmit antenna gain: $-10 \mathrm{dBi}$

Receive antenna (G/T): $20 \mathrm{~dB} / \mathrm{K}$

C/No for $2500 \mathrm{~km}: 67 \mathrm{dBHz}$

Channel symbol rate (half rate coding): 2 Mbps

Coding gain (turbo coding): $8.0 \mathrm{~dB}$

$E_{\mathrm{b}} / N_{0}: 15.589 \mathrm{~dB}$. 


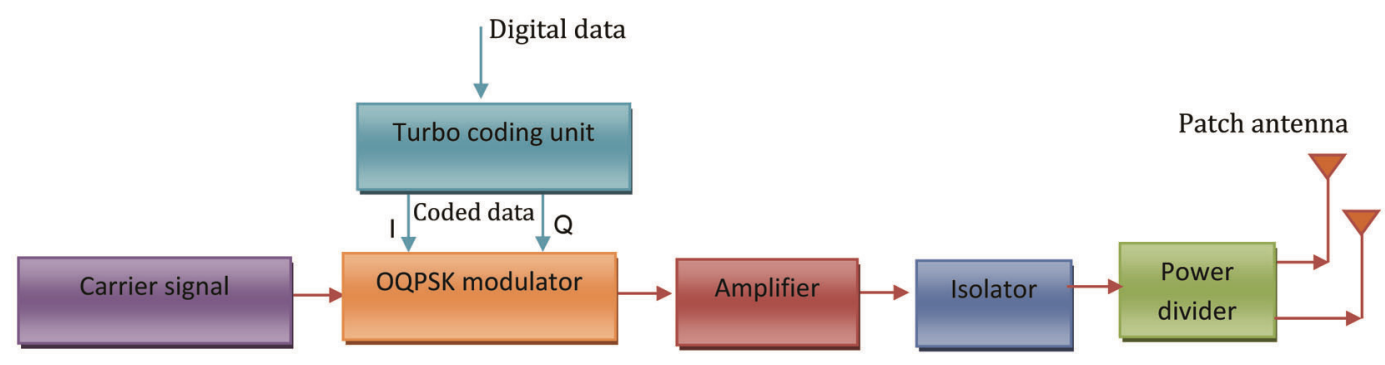

Figure 4. Block diagram of digital transmitter with direct modulation.

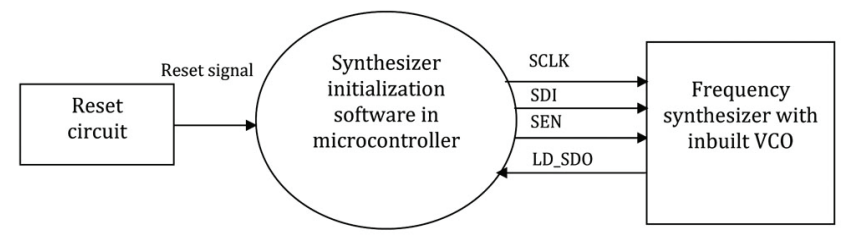

Figure 5. Interface diagram of carrier generation circuit.

\section{Carrier generation Technique}

Carrier frequency can be generated using different techniques. However, generating high frequency with frequency agility and high-resolution capability is the need of this modern electronic age. Hence in digital telemetry transmitter, carrier generation is being implemented using frequency synthesizer technique. In addendum to this, to bring functional integration in the system, a frequency synthesizer with in-built Voltage Controlled Oscillator (VCO) is selected. This offers low component count, high packing density, and ease of programmability and flexibility.

The frequency synthesizer is a device (an electronic system) that generates a large number of precise frequencies from a single reference frequency. A low noise, wide band, fractional- $N$ phase-locked-loop (PLL) with an integrated VCO having a fundamental frequency of 1.5$3 \mathrm{GHz}$, and an integrated $\mathrm{VCO}$ output divider that together allow the synthesizer to generate frequencies from $10 \mathrm{~s}$ of $\mathrm{MHz}$ to $3 \mathrm{GHz}$. The integrated phase detector and delta-sigma modulator permit wider loop bandwidths with excellent spectral performance. A suitable synthesizer with better phase noise and spurious performance across all frequencies is selected so as to enable it to provide excellent transmitter spectral purity. The fractional- $N$ synthesizer has the ability to decrease phase noise, provide increased loop speed for a given step size and provide reduced reference spur levels.

The design of the carrier generation at $2259 \mathrm{MHz}$ is discussed below. TCXO of $40 \mathrm{MHz}\left(F_{\mathrm{OSC}}\right)$ with $1 \mathrm{ppm}$ stability is taken as the reference input to which VCO will be locked to the desired frequency. Phase detector frequency $\left(F_{\mathrm{PD}}\right)$ is taken as $40 \mathrm{MHz}$ so that it offers better step size, phase noise and switching speed. $R=F_{\mathrm{OSC}} / F_{\mathrm{PD}}$.
Hence reference counter, $R=1$ and feedback counter, $N=F_{\mathrm{VCO}} / F_{\mathrm{PD}}$. To generate $2259 \mathrm{MHz}, N=56.475$. Thus integer $=38_{\mathrm{h}}$, fraction $=79999 \mathrm{~A}_{\mathrm{h}}$.

To initialize the synthesizer, an 8-bit microcontroller and its associated reset circuit are used. As TCXO employs a crystal, the synthesizer subsystem is mounted on vibration isolators to avoid any piezoelectric effect during vibration. Hence the system offers better phase noise even under high dynamic conditions of LV. Figure 5 shows the block diagram and interfaces of the carrier generation circuit. Figure 6 shows the phase noise plot at $2259 \mathrm{MHz}$.

\section{Modulation}

OQPSK modulation is selected as it offers use of less linear amplifiers. Maximum phase transition is $90^{\circ}$, thus preventing signal flow through the origin ${ }^{1}$. This is implemented using S-band IQ modulator which enables direct modulation in S-band carrier signal making the system less complex and reliable. An IQ modulator with excellent phase accuracy and amplitude balance is selected for direct modulation to RF. The carrier signal is fed differentially to provide better performance in carrier suppression/local oscillation (LO)-feed-through.

The non return to zero (NRZ) message signals ( $I$ and $Q)$ are driven by a line driver from the baseband system and are received at the modulator using a line receiver. To prevent spectral splatter, premodulation filters are used in both the channels. Baseband signal amplitude and bias are adjusted for optimum performance. These are further split into differential signals using differential driver for driving the modulator. Figure 7 shows the S-band modulated output.

\section{System performance in Crew Escape System}

The Crew Escape System (CES) in HSP missions provides reliable escape provisions for the crew during ascent phase of LV, which consists of quick-acting solid motors that take the Crew Module (CM) to safety in case of any exigency at the launch pad or during ascent phase of LV. Performance of CES and its capability to execute 

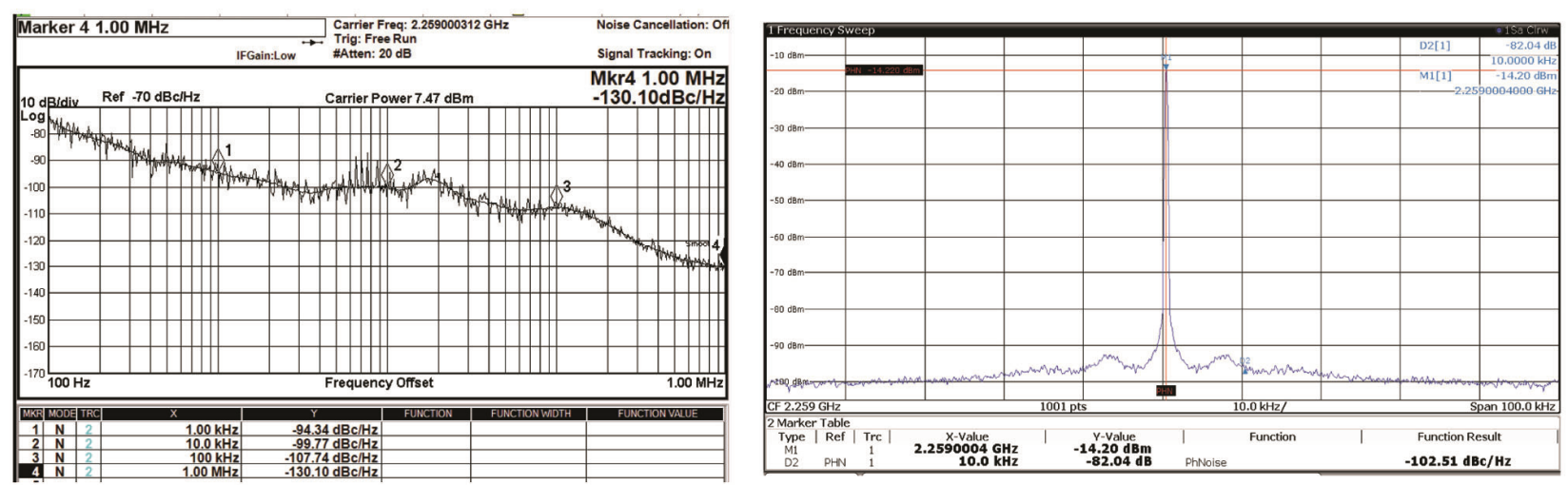

Figure 6. Phase noise and carrier plot.

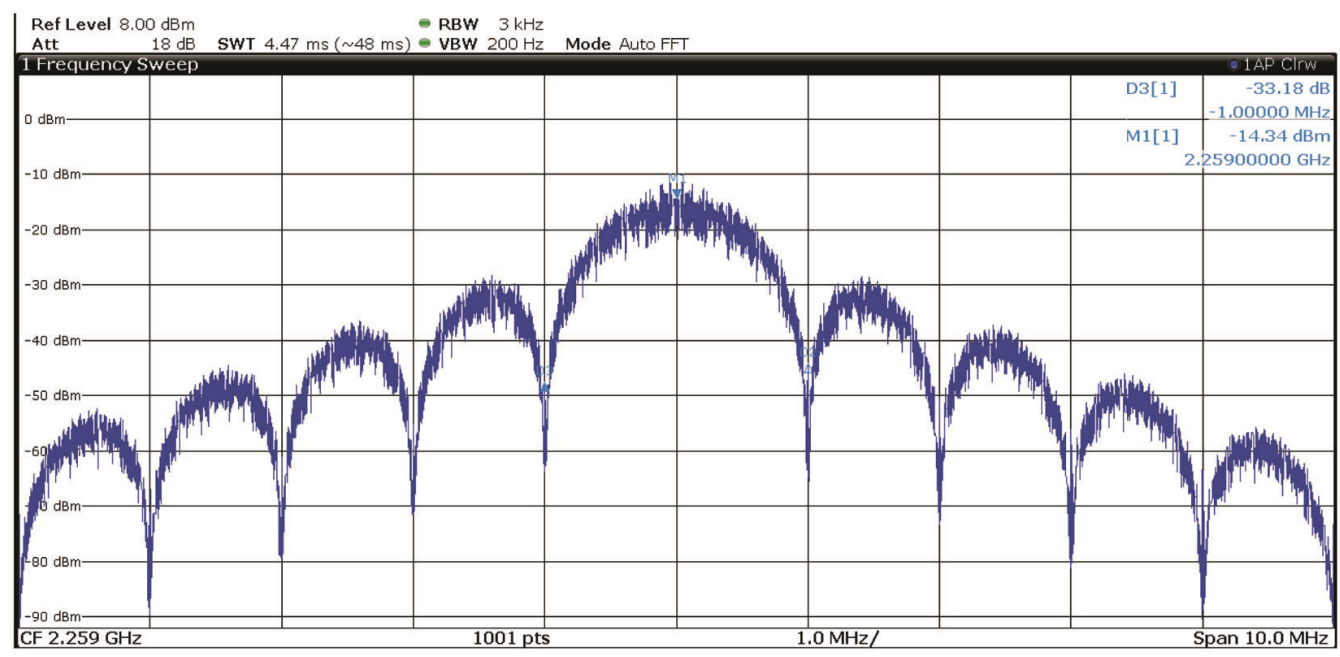

Figure 7. S-band modulated output spectrum.

a ground-based abort were demonstrated through PAT flight. Digital telemetry transmitter operating in the S-band was flown in the CES to provide telemetry data of the mission in real time.

\section{Digital telemetry transmitter}

The digital telemetry transmitter is a $0.5 \mathrm{~W}$ OQPSK transmitter in which modulation is performed directly in the S-band at $1 \mathrm{Mbps}$ data rate with half rate turbo coding in the baseband level, which gives $2 \mathrm{Mbps}$ symbol rate. It performs CCSDS recommended rate half turbo coding of telemetry data to improve telemetry link margin, resulting in reduction of transmitter power. It is a high-performance, forward error correction code and provides a coding gain of $9 \mathrm{~dB}$. Coding helps to closely approach channel capacity.

The system is capable of operating at any one of the pre-assigned S-band telemetry frequencies (2211/2237.5/ $2259 / 2275 \mathrm{MHz}$ ). This is possible using microcontroller selection logic. The $2259 \mathrm{MHz}$ frequency was assigned to the digital telemetry transmitter in this mission based on interference study with other systems in PAT. It has an in-built DC/DC converter.

Two identical patch antennas mounted diametrically opposite on $\mathrm{CM}$ were used to provide omnidirectional coverage and fed using a Wilkinson S-band power divider. This consists of two rectangular microstrip patches with linear polarization. It is fabricated on RT Duriod 6002 material with a dielectric constant of 2.94 . The antennas are capable of withstanding temperatures up to $200^{\circ} \mathrm{C}$. Figure 8 shows the complete digital telemetry transmitter system.

The system performance was evaluated at designer level and subjected to test and evaluation according to the ETLC levels of the PAT mission like hot, cold, vibration, vacuum and EMI. The system was integrated in the Avionics bay of PAT and radiation tests were conducted. System integrity was evaluated by implementing reradiating set-up and reception of data at the ISTRAC ground station. Suitable amplifiers was inserted in the chain to meet the link requirement in the reradiating set-up. 


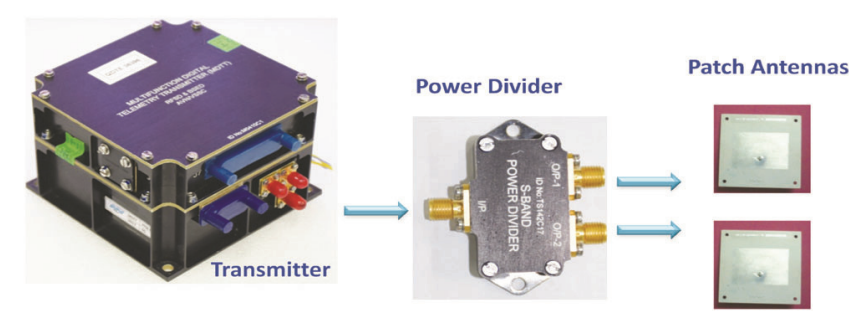

Figure 8. Digital telemetry transmitter system.

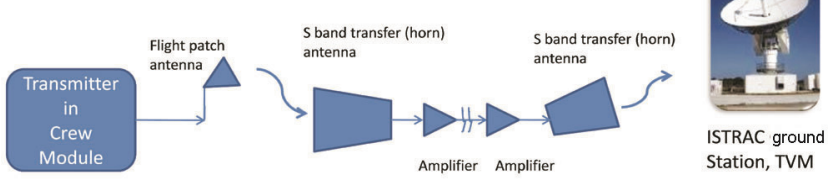

Figure 9. Reradiating set-up used in the integrated level test.

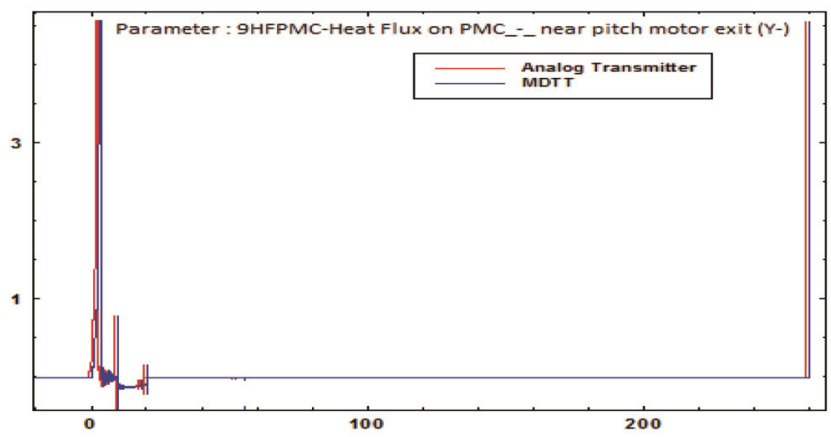

Figure 10. Typical parameter plot comparison between analog and digital transmitters.

Figure 9 shows the reradiating set-up. Demodulation of transmitter signal and decoding of the data are executed using the ground station receiver.

System integrity was validated till the launch phase by employing reradiating set-up according to the location of the ground station and the distance involved between the transmitter and receiver.
For the entire integrated test at the launch centre and on the day of launch three ground stations, viz. SHAR-1, SHAR-2 and SHAR-3 were deployed to receive the telemetry (TM) data. The package was powered on from $T_{0}-30 \mathrm{sec}$ to $T_{0}+259 \mathrm{sec}$. Data acquisition through the ground stations started from $T_{0}-8 \mathrm{sec}$. Package performance was normal throughout the mission. All the parameters received through digital transmitter in HSP-PAT were compared with regular telemetry data and found to be normal. Figure 10 shows a typical parameter plot received through MDTT as TM3 and normal analog telemetry (TM2). A shift of $1 \mathrm{sec}$ is given intentionally for easy interpretation of match between the two systems.

\section{Conclusion}

A digital telemetry transmitter with OQPSK direct RF modulation and turbo coding is realized and experimented in CES. OQPSK modulation enables the system to use less linear amplifier with minimum spectral occupancy and $\mathrm{ISI}^{4}$. Direct RF modulation offers a simple compact system with minimum component count and better reliability. Coding gain offered by turbo coding in the baseband system reduces the high RF transmit power requirement for establishing the link for a particular data rate. With the above features, the system performed well in the PAT mission and provided data for the entire duration of the mission.

1. Haykin, S., Digital Communication, Wiley Press, 2013.

2. Lathi, B. P., Modern Digital and Analog Communication Systems, Oxford University Press, New Delhi, 3rd edn.

3. Carlson, A. B., Crilly, P. B. and Rutledge, J. C., Communication Systems, McGraw-Hill, Singapore, International Edition, 2002, 4th edn.

4. Gronemeyer, S. and McBride, A., MSK and offset QPSK modulation. IEEE Trans. Commun., 1976, COM-29(8), 809-819.

doi: $10.18520 / \mathrm{cs} / \mathrm{v} 120 / \mathrm{i} 1 / 161-165$ 